\title{
Hepatitis $C$ quasispecies adaptation in the setting of a variable fidelity polymerase
}

\author{
This article was published in the following Dove Press journal: \\ Virus Adaptation and Treatment \\ 23 July 2012 \\ Number of times this article has been viewed
}

\section{Daniel Schmidt-Martin' \\ Orla Crosbie ${ }^{2}$ \\ Elizabeth Kenny-Walsh ${ }^{2}$ \\ Liam J Fanning'}

'Molecular Virology Diagnostic and Research Laboratory, Department of Medicine, University College Cork, Clinical Sciences Building, Cork University Hospital, ${ }^{2}$ Department of Gastroenterology and Hepatology, Cork, Ireland
Correspondence: Daniel Schmidt-Martin Molecular Virology Diagnostic and Research Laboratory, Department of Medicine, University College Cork, Clinical Sciences Building, Cork University Hospital, Cork, Ireland

Tel +3532 2I 490 I226

Fax +353 2I 490 I227

Email d.schmidt@ucc.ie

\begin{abstract}
Hepatitis C (HCV) is a virus characterized by an RNA-dependent RNA polymerase that lacks a proofreading mechanism and, as a result, generates a quasispecies. There is emerging evidence that this RNA-dependent RNA polymerase may in fact have variable fidelity. Here, we review the relevant concepts, including fitness landscapes, clonal interference, robustness, selection, adaptation, mutation rates, and their optimization, and provide a unique interpretation of a number of relevant theoretical models, evolving the theory of replicative homeostasis in light of their findings. We suggest that a variable fidelity polymerase can find its own optimal mutation rate, which is governed by the sequence itself and certain population dynamics. We propose that this concept can explain features of viral kinetics and clearance, both spontaneously and following treatment of chronic HCV. We point to evidence that supports this theory and explain how it refines replicative homeostasis and conclude by discussing particular areas of potential research that might augment our understanding of viral host interactions at an individual cellular level.
\end{abstract}

Keywords: fitness landscapes, adaptation, evolution, quasispecies, hepatitis $\mathrm{C}$, replicative homeostasis

\section{Introduction}

Hepatitis C (HCV), a positive 9.2-9.6 kb RNA Flavivirus, was first identified by Choo and colleagues at Chiron in 1989 and is estimated to infect up to $3 \%$ people worldwide, equivalent to $120-170$ million people. ${ }^{1-3}$ Chronic HCV infection leads to the development of cirrhosis in $20 \%$ of cases after 20 years and is now the leading indication for orthoptic liver transplantation in the USA.

Low fidelity and the lack of proofreading ability of the HCV RNA-dependent RNA polymerase (RDRP) results in a population of closely related genomes or quasispecies. ${ }^{4}$ Originally proposed by Eigen as a model for the study of the evolution of primitive organisms, the quasispecies concept has been applied to many bacteria and viruses including human immunodeficiency virus and $\mathrm{HCV}^{5}$ The gradual generation of point mutations results in the development of new variant species or "quasispecies" with slightly altered characteristics that then undergo selection. Within a given host, those quasispecies best adapted to the environment are most likely to survive and become dominant as a result of the principle of competitive exclusion. ${ }^{6}$ The most prevalent quasispecies is the "master" sequence and other related quasispecies cluster around this in terms of their genetic distance. Mutations either undergo selection (positive, resulting in the selection of beneficial traits; negative, when a deleterious trait is removed). Alternatively, in the absence of selection, the gradual accumulation of 
neutral or near neutral mutations of insufficient magnitude to prompt selection results in an evolutionary process known as "genetic drift." Gradual adaptation to the host occurs as a result of these processes with the neutral theory of evolution predicting that genetic drift will be the predominant form of evolution. ${ }^{7}$ For a mutation to provoke a change from genetic drift to natural selection it must breach what has been termed the "selection threshold."

These processes are dependent on a number of factors including virion fitness, population size, clonal density, clonal interference, and mutation rates. Our understanding of HCV has grown exponentially as a result of both experimental results and mathematical modeling, which have facilitated better understanding of viral replication processes and, as a result, viral genomic selection, adaptation, and evolution. This review provides an up-to-date appraisal of these topics.

\section{Fitness}

Conceptually, each genome has an inherent fitness defined by a group of characteristics (ability to infect, ability to replicate, energy requirement), with each quasispecies competing for host resources (host cells, cellular machinery, etc). Within the population, each sequence competes for these host resources with the best adapted, or fittest characteristics, most likely to dominate. However, the transience of this domination is guaranteed by the mechanism by which it is generated; the almost inexorable emergence of fitter mutants demands continual evolution for survival, in a process called the "Red Queen Hypothesis." "A moderate increase in viral fitness of one quasispecies over another results in exponential proliferation of this new quasispecies, with likely extinction of its competitor sequences in what amounts to a zero-sum game. ${ }^{10}$

\section{Fitness landscapes}

Viral fitness can be described in the form of a fitness landscape, with mountains corresponding to areas of increased fitness surrounded by areas of diminishing fitness analogous to foothills (Figure 1). The accumulation of mutations allows the exploration of the sequence space and through this process the discovery of fitness gains that might displace the master sequence through competition. In the case of $\mathrm{HCV}$, because the number of possible nucleotide combinations is so great $\left(4^{9.600}\right)$, this landscape is only able to describe quasispecies diversity for short segments of the sequence. Not all mutations are viable, with these lethal mutations akin to cliffs in what is known as a "truncated fitness landscape." Finally, the combined interplay between individual quasispecies and the

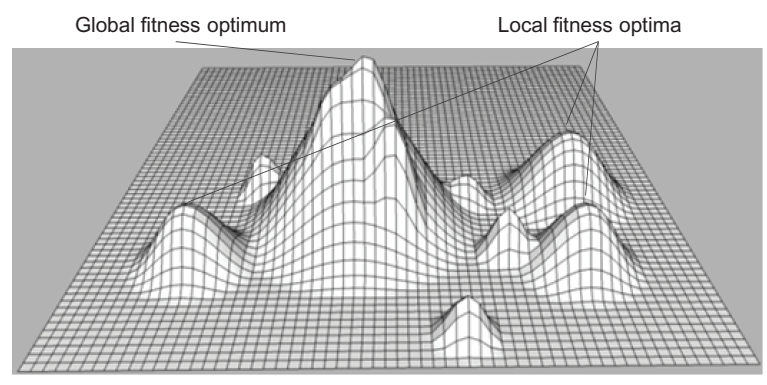

Figure I Schematic representation of the sequence space in the form of a fitness landscape.

Notes: The accumulation of mutation facilitates the exploration of the landscape. Adaptation results in the discovery of local fitness optima and potentially the global fitness optimum.

immune system results in a changing or dynamic truncated fitness landscape.

In this setting, the lack of a proofreading function is often looked upon as beneficial to HCV; adapted mutants, which are closely related to the parent virion and better able to evade the host's immune response, emerge and maintain chronic infection. However, in this model the proviso is that high mutation rates mean that beneficially adapted mutants are equally prone to deleterious mutations, which can potentially wipe out entire quasispecies. Muller's ratchet predicts that deleterious mutations are likely to "hitchhike" and be found in all future progeny, barring the unlikely event of a reciprocal mutation taking place. ${ }^{11,12}$ Mitigating the effects of hitchhiking is the process of recombination, which can facilitate the removal of deleterious mutations by combining mutation-free segments and allow greater potential exploration of the sequence space by combining sequences with multiple mutations. It is this latter process that is thought to contribute significantly to the emergence of differing HCV genotypes and even taxa and species. ${ }^{13,14}$

\section{Interference}

Although a significant factor in determining the fate of a given quasispecies, competitive exclusion is not the sole determinant of evolutionary success. In large quasispecies populations, it has been shown that sequences with significant fitness superiority are not necessarily guaranteed to dominate a quasispecies due to a process known as "clonal interference." ${ }^{15-17}$ In small populations, beneficial mutations of smaller increments are more likely to come to dominate as a result of selective sweep, while, in large populations (as are seen in established chronic HCV infections), a quasispecies with significant fitness benefit can be suppressed by the less-fit dominant quasispecies, unless it reaches a 
critical threshold. This has the net effect of ensuring that, in chronic infection, the incremental increase in quasispecies fitness becomes larger in fitness gain but more infrequent in occurrence. Experimental evidence of clonal interference supporting this theory has been found in Escherichia coli, DNA viruses, $\mathrm{HCV}$, and the RNA vesicular stomatitis virus (VSV). ${ }^{16,18-21}$

\section{Defective interfering particles}

Notwithstanding the extreme variability seen in the genetic sequence of RNA viruses, it must be remembered, however, that redundancy in the sequence is limited and that the proteins produced are small in number and, in most cases, essential in function. However, despite this lack of redundancy, subgenomic particles exist that can have significant effects on virus population dynamics.

Named "defective interfering particles" (DIPs) and identified in several virus species (including both DNA and RNA viruses), they may be important factors in the search for fitter quasispecies resistant to the effects of DIPs. ${ }^{22-26}$ Unable to replicate in the absence of wild-type virus but able to infect new cells, they are thought to contribute to the oscillating nature of the viral load repeatedly seen in HCV infection. DIPs have also been proposed to interfere in the production of wild-type virus and modulate pathogen virulence and may themselves be potential antiviral agents. ${ }^{27-30}$

Stumpf and Zitzmann have proposed the reciprocality of DIPs; that is, that the particles are able to replicate but are unable to cause de novo infection of new cells due to the deletion of the structural section of the genome. The associated increase in replicative ability leads to competitive exclusion of viable virions and the gradual accumulation of defective intracellular viral RNA, meaning that continuous de novo infection of new cells is essential to viral survival. ${ }^{31}$ Experimental evidence for this has remained elusive.

Studies focused on hepatocyte-derived HCV genomic sequences have not found evidence of these particles, though factors such as the duration of infection and use of limited numbers of clones (it is estimated that use of 20 clones will demonstrate most sequences present at a level of $10 \%$ ) may go some way to explain this. ${ }^{32-34}$ Indeed, the advent of next-generation sequencing may see the reemergence of this concept.

\section{Robustness}

The ability of a virion to tolerate mutations without phenotypic disruption, termed "robustness," is also likely to be important in maintaining or enhancing fitness.
Characterized by a greater number of available neutral mutations, a high degree of robustness results in a smoother fitness landscape, in a theory described as "survival of the flattest." 35 Studies using digital models and subviral particles suggest that an organism with greater robustness may out compete and dominate less robust counterparts, particularly at times of high mutagenesis. ${ }^{35-37}$

The emergence of neutral mutation-rich organisms may however have significant implications for virion evolution. A recent study has demonstrated that a high proportion of neutral or near-neutral mutations may act as a barrier to evolution by natural selection, with genetic drift coming to dominate. ${ }^{8}$ Studies evaluating HCV robustness are limited, but one network-based analysis of HCV polyprotein has demonstrated a high degree of robustness at many nucleotide positions, with relatively few positions vulnerable to phenotypically deleterious mutation. ${ }^{38}$ Comparisons with other RNA viruses are challenging, as direct studies have not yet been published. One recent paper has estimated by site-directed mutagenesis that $40 \%$ of random mutations in VSV are lethal, which may suggest a lesser degree of robustness compared with $\mathrm{HCV}^{39}$

Finally, it has been suggested that increased robustness may result in a reduced ability to adapt and that, in organisms that are required to survive in changing environments, the requirement for frequent adaptive change will limit tolerance of neutrality/robustness. ${ }^{40}$ Indeed, the ability of an organism to respond to selective pressure and tolerate significant large-scale genetic evolution or evolvability has also been demonstrated as a selectable trait. ${ }^{41}$

\section{Cooperative interaction}

The concept of the "cooperative interaction" of the constituent mutants in exploring fitness maxima, so that the population ultimately achieves a mutation-selection equilibrium, distinguishes quasispecies theory from classical population genetics. When looked on in this light, it becomes apparent that successful quasispecies evolution is a population-wide phenomenon, so that fitness can be seen as an "ensemble property." ${ }^{22}$ While evidence for this phenomenon is limited, studies of poliovirus have demonstrated that the pathogenesis of individual quasispecies is affected by cooperative interaction with other mutants in the quasispecies profile and that maintenance of a degree of heterogeneity is preferable for viral survival and maintenance of tissue tropism. ${ }^{43}$ Indeed, the influence of cooperative reactions has been proposed as essential if mathematical models are to accurately generate the quasispecies patterns observed in vivo. ${ }^{44}$ 


\section{Adaptation}

"Adaptation" is the process whereby the quasispecies alters to become more suited to new or changing environments. The rate of adaptation of quasispecies appears to be governed by a number of factors: population size, mutation rate, adaptive quotient, and variability of the environment. In small populations, the size of the population limits the ability to explore the sequence space. As a result, adaptation occurs at a slower rate by means of stochastic genetic drift with episodic selective sweeps. This means that the population is more likely to be confined to local fitness peaks. In contrast, large populations are better able to expand throughout the sequence space and, as a result, adaptation is more deterministic, though the time taken for fixation of beneficial mutations is increased as a result of interference..$^{45,46}$

\section{Mutation rates}

The effects of different mutation rates on a quasispecies within a truncated fitness landscape appear to follow three patterns: (1) low mutation rates result in a distribution around the master sequence and are more likely to become "trapped" in local fitness peaks, reducing the chances of complete exploration of the sequence space; (2) intermediate mutation rates result in wider exploration of the sequence space, with the emergence of variants further removed from the master sequence; and (3) those with high mutation rates produce an ever-increasing number of progeny with lethal mutations and, as a result, reach what has been called the "error threshold" - the point at which the quasispecies becomes unable to maintain sequence integrity. The coercion of viruses beyond this point into what has been called "error catastrophe" has been a major strategy in the development of antiviral therapies. ${ }^{46-49}$

Interestingly, adaptation is not maximal prior to reaching error threshold; rather, it behaves in a sine wave fashion (Figure 2). The mutation rate that results in optimal adaptation is remote from error threshold and adaptation decreases with increasing mutation rate as the ability to fix beneficial mutations decreases until error threshold is breached. ${ }^{50,51}$ Using parameters present in quasispecies, Orr has found that optimal mutation rates for adaptation are governed by the strength of selection against deleterious mutations (ie, more truncated landscapes have a lower optimal adaptive mutation rate). ${ }^{50}$ This work was based on the assumption that the selective power against deleterious mutations was at all times greater that the selective power for beneficial mutations; however, this may not, in fact, be the case.

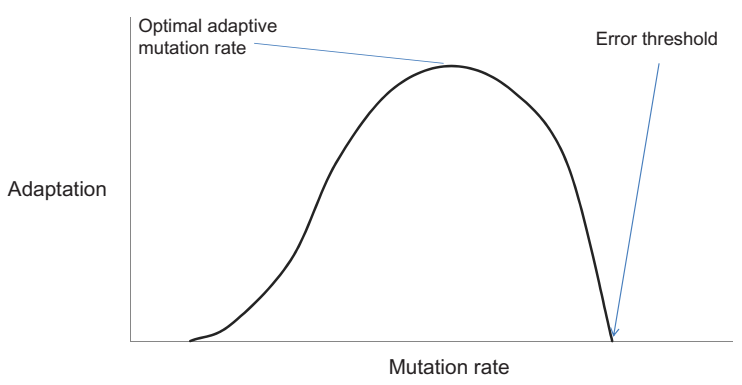

Figure 2 The interaction between mutation rates and the rate of adaptation. Notes: Beyond the optimal adaptive mutation rate, increasing mutation rates result in an ever-diminishing rate of adaptation until the error threshold is reached.

\section{Adaptive quotient}

Johnson and Barton advanced this theory by describing a matrix that can predict a sequence-specific optimal mutation rate depending on whether the surrounding fitness landscape is dominated by deleterious or beneficial mutations and the selective power of these relative to each other. ${ }^{52}$ According to this model, the existence of many potential beneficial mutations will promote the emergence of a higher mutation rate and vice versa. In the setting of a bottleneck event (rapid reduction in quasispecies - as occurs at transmission of HCV) the organism can be seen to be less adapted to the new host and, as a result, the ratio of beneficial:deleterious mutations is also likely to change and will probably be reflected in the rate of mutation..$^{53}$

\section{Variability of environment}

In static environments, the exploration of the sequence space with fixation of beneficial mutations that pass the selection threshold and outlast clonal interference becomes exhausted once the quasispecies reaches the mutation-selection equilibrium. At this stage, all fitness optima have been explored. As this occurs, the fitness gains that were initially large, diminish toward nil. ${ }^{54}$ With many microorganisms, however, the emergence of new environments, either as a result of transmission of infection or the development of immune responses, results in a dynamic fitness landscape that serves to replenish the potential for adaptive change.

Furthermore, the ruggedness of these landscapes can themselves affect the rate of adaptation. Clune et al demonstrated using computer models that digital organisms fail to optimize mutation rates and tend to settle at a mutation rate below this. ${ }^{55}$ Evidence for this has been described in DNA bacteriophages, where the imposition of a fourfold increase in mutation rate actually conferred fitness gain. ${ }^{55,56}$ Clune et al argued that, while adaptation occurs over long periods (many generations), selection acts quickly and this phenomenon 
may be an effort by the virus to mitigate the potential for emergence of deleterious/lethal mutations. Expanding on this initial finding, Clune et al demonstrated that the observed mutation rate is dependent on the ruggedness of the fitness landscape with more rugged landscapes favoring an even lower mutation rate..$^{55}$

\section{The fidelity spectrum}

There is a growing body of literature indicating that mutation rates are not constant and may be selectable. Mutation rates have been shown to increase at times of stress in many bacteria and lethal mutagenesis has long been suggested as a potential treatment strategy in viral infections. ${ }^{57}$ The beneficial effects of increased mutational rates, in addition to how they may be associated with increased replicative capacity, have also been demonstrated in bacteriophage populations ${ }^{58}$ Furthermore, adaptive change in the mutation rate in response to medications has been shown to confer drug resistance and sustain chronic infection in the case of human immunodeficiency virus type $1 .{ }^{59}$ Several mechanisms governing how transient increases in mutation rate can be generated and suitably regulated have been suggested, including environmental and heritable factors. ${ }^{60}$

\section{Evidence of variable RDRP mutation rates in $\mathrm{HCV}$}

In $\mathrm{HCV}$, the estimated mutation rate is $1 \times 10^{-4}$ to $5 / \mathrm{base}^{61-63}$ The estimation of the error threshold of HCV RDRP is $10^{-2}$ to 3 (mutations per base), which leaves scope for a ten- to hundredfold change in baseline RDRP fidelity before the error threshold is reached, with the optimal adaptive mutation rate likely to be found within this range. Lethal mutagenesis has formed one of the theories of the mechanism of action of ribavirin, as it has been shown to induce lethal mutagenesis in poliovirus and foot-and-mouth disease, but the results in HCV have been variable. ${ }^{62,64,65}$ Ribavirin-resistant mutations have been described, both in vitro and in vivo, in two HCV nonstructural proteins (NS5A and NS5B) including the RDRP. It has been suggested that the NS5A mutation may indicate that this protein may interact with the RDRP to modulate polymerase fidelity. ${ }^{62,66,67}$ Indeed, the idea that RDRP fidelity may be controlled remotely is not novel to $\mathrm{HCV}^{68}$

Recently, mutant RDRPs conferring ribavirin resistance by means of increased fidelity have been described in both foot-and-mouth disease virus and poliovirus. We feel that similar mutants are likely to exist in the case of HCV and that their emergence during ribavirin therapy would explain the heterogeneity of the effect on mutation rates seen in these studies. Furthermore, the sampling intervals may have been such that transient increases in mutation rates may have been missed.

A mechanism by which viruses might self-regulate replication fidelity has been proposed by Sallie in his theory of replicative homeostasis (RH). ${ }^{69-71}$ Sallie argued that HCV viral kinetics behave in such a way to suggest autoregulation of virion production through a homeostatic mechanism that modulates RDRP fidelity/processivity (which he proposed are inversely proportional). RH predicts that excess wildtype protein will prompt a decrease in fidelity and a resultant increase in mutation and vice versa. The idea that mutation rates may be dependent on polymerization rates was first proposed in the kinetic proofreading hypothesis, in which a delay in the rate of polymerization results in increased polymerase fidelity; experimental support for this has been demonstrated in the case of VSV. ${ }^{11,72}$ While we are of the opinion that Sallie's theory has significant merit, we feel that the theory of RH could be further adapted to more accurately describe the behavior of $\mathrm{HCV}$.

\section{A framework for the action of a variable fidelity polymerase}

On the basis of the position of the quasispecies within the fitness landscape, we propose that a framework exists for the selection of phase-specific mutation rates (Figure 3). The RDRP acts along a fidelity spectrum with optimal mutation rates that are largely dependent on population size, capacity for adaption (adaptive quotient), and variability of

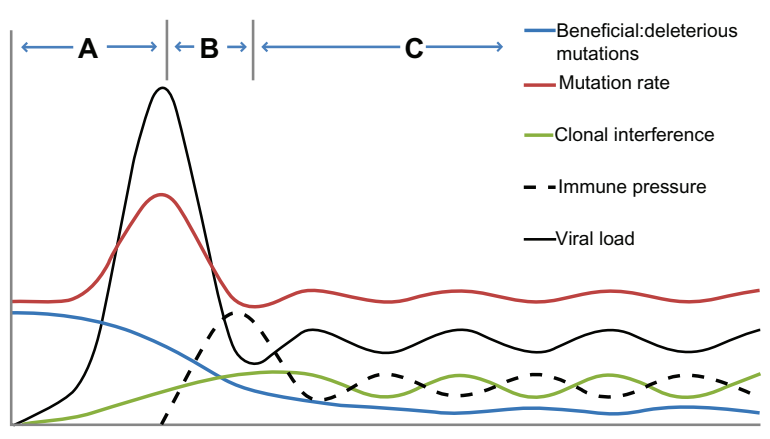

Figure 3 Phase diagram demonstrating the proposed behavior of hepatitis $\mathrm{C}(\mathrm{HCV})$ variable fidelity polymerase. (A) New infection is characterized by an increase in the ratio of beneficial:deleterious mutations. HCV polymerases with increased mutation rates are selected, promoting exploration of the sequence space, which results in viral load spike. (B) Once the sequence space is explored, the ratio of beneficial:deleterious mutations decreases and the polymerase mutation rate returns to baseline. As the quasispecies expands, clonal interference emerges. The advent of host immune response, in combination with reduced mutation, is associated with a marked reduction in viral load. (C) Immune-mediated dynamic changes in the fitness landscape result in oscillation of clonal interference, viral load, and polymerase mutation rates. 
the environment. ${ }^{73,74}$ We propose that the optimal mutation rate selected for could be predicted by the position of the sequence within a framework similar to that proposed by Johnson and Barton. ${ }^{52}$ The exploration of this fidelity spectrum is likely to be initially stochastic, as it is reliant on the generation of promutator mutations and evidence for similar processes can be seen in Drosophila populations. ${ }^{52,75}$

Following the bottleneck of transmission, unencumbered by clonal interference, and with an increased probability of beneficial mutations, we suggest that a form of densitydependent selection, similar to those that have been described in foot-and-mouth disease virus, E. coli, and Drosophila, will result in the emergence of an increased mutation rate. ${ }^{75-77}$ This latter occurrence would be characterized by quicker adaptation, could correspond to the intermediate fidelity phase as described by Saakian et al, and could be likened to the episodes of stress which have also been shown to result in increased mutation rates in E. coli. ${ }^{47,78}$ As the relative proportion of beneficial to deleterious mutations is increased in small nonadapted populations, the emergence of an increased mutation rate is favored. Initial infection with a finite number of variants will gradually explore local fitness maxima by stochastic means until the population becomes sufficient for deterministic exploration as the capacity to generate all possible mutants is achieved. ${ }^{46}$ With population expansion, increasing clonal interference, and viral adaptation, the same process will select a less-productive polymerase with increased fidelity that has the added potential bonus of being immunologically stealthy by means of viral-load reduction. This period of selection may result in the reduction in viral load often seen in acute $\mathrm{HCV}^{70}$

Furthermore, increased fidelity will inevitably mean that antigenic thresholds will be intermittently breached, resulting in activation of the adaptive immune response. As the exploration of both sequence space and what we refer to as the "fidelity spectrum" is stochastic, it is to some degree dependent on chance, but the near certainty of successful exploration has been in built into the quasispecies characteristics of the virus. Conversely, the certainty of failure, in some cases, to either optimize fidelity or even find infidelity sufficient to evade the immune response, provides the tantalizing prospect of explaining the process by which infection is cleared in $15 \%-25 \%$ of patients. ${ }^{79,80}$ The emergence of a population selection-mutation equilibrium will tend toward a lower mutation rate, as the genetic distance to the nearest beneficial mutations is likely to become larger due to this adaptation. In summary, at times of stress, the polymerase and its inherent mutation rate becomes the unit of selection, while, at other times, it is the genomic properties and their cooperative/competitive interactions that become the traits selected for or against.

In our model, similar to that of $\mathrm{RH}$, the selection of particular sequences for removal by the immune system will merely result in the generation of new quasispecies to match the new fitness landscape, while also facilitating longterm stability of quasispecies in the absence of variations in effective immune pressure. This model also has the capacity to explain HCV clearance in the absence of seroconversion, as it allows for the attainment of error catastrophe without the need for immune response. Additionally, our proposed mechanism of action along a fidelity spectrum more coherently explains why the emergence of a single dominant quasispecies in the treatment of $\mathrm{HCV}$ infection and a low rate of quasispecies evolution are more likely to result in clearance as opposed to the generation of new quasispecies, as Sallie's model would suggest. ${ }^{69-71}$

In proposing this model, we must acknowledge that one of the major obstacles to clarifying the interaction between quasispecies theory and experimental results in $\mathrm{HCV}$ is the phenomenon of founder effect at the level of the individual cell. The prevention of superinfection, in theory, means that the apparatus of the cell is at the mercy of this sole founder and that competition is prevented, promoting the preservation of the status quo. This, coupled with evidence demonstrating the prevention of infection of neighboring cells via the apical cell membrane and the facilitation of virion transfer to these neighbors via tight junctions, is equivalent to dynasty building - that is, clonal expansion. Accounting for these factors in evolutionary models is challenging, particularly when little is known of the incidence of superinfection in the context of fitter "pilgrim" virions, which may facilitate the conversion of the quasispecies to new fitness optima.

Finally, we would like to note one conundrum reconciling the current theories of optimal mutation rates and the suggestion that organisms adapt toward neutral networks. Under the survival of the flattest hypothesis, the emergence of such fitness landscapes results in a reduction in the ruggedness of the fitness landscape. As the number and selective power of potentially deleterious mutations are reduced, we should see closer optimization of mutation rates to maximize adaptation. However, little evidence has been produced to favor this and, conversely, the mutation rate in E. Coli, which has a $90 \%$ tolerance of mutations, has a mutation rate far less than that of RNA viruses, which have a lethal mutation rate of $21 \%-40 \%{ }^{39,81}$ 


\section{Conclusion}

Adaptive evolution is slave to both genetic drift and natural selection, with the emergence of more neutral flatter fitness landscapes favoring the former. Following a bottleneck, the exploration of the sequence space is stochastic, with the transition to deterministic exploration dependent on the population size and the development of clonal interference. Mutation rates often fail to optimize adaptation and this may be an effort to mitigate the relative strength of lethal mutations when compared with the relative and often-marginal benefit of beneficial mutations - particularly, in well-already-adapted species. Mutation rates are not constant and, in low population sizes, increased mutation rates may be selected for to enhance the rate of adaptation. Several potential mechanisms for regulating mutation rates to ensure that these increases are transient have been proposed. HCV demonstrates characteristics consistent with a population density-mediated selection of mutation rates.

\section{Acknowledgment}

The authors would like to acknowledge the support of Daniel Schmidt-Martin from Molecular Medicine Ireland.

\section{Disclosure}

The authors report no conflicts of interest in this work.

\section{References}

1. Choo QL, Kuo G, Weiner AJ, Overby LR, Bradley DW, Houghton M. Isolation of a cDNA clone derived from a blood-borne non-A, non-B viral hepatitis genome. Science. 1989;244(4902):359-362.

2. Alter MJ. Epidemiology of hepatitis C virus infection. World $J$ Gastroenterol. 2007;13(17):2436-2441.

3. Pockros PJ. Developments in the treatment of chronic hepatitis C. Expert Opin Investig Drugs. 2002;11(4):515-528.

4. Steinhauer DA, Domingo E, Holland JJ. Lack of evidence for proofreading mechanisms associated with an RNA virus polymerase. Gene. 1992;122(2):281-288.

5. Eigen M. Selforganization of matter and the evolution of biological macromolecules. Naturwissenschaften. 1971;58(10):465-523.

6. Gause GF. The Struggle for Existence. New York, Dover; 1971.

7. Kimura M. The Neutral Theory of Molecular Evolution. Cambridge: Cambridge University Press; 1983.

8. Nelson CW, Sanford JC. The effects of low-impact mutations in digital organisms. Theor Biol Med Model. 2011;8:9.

9. Van Valen L. A new evolutionary law. Evol Theory. 1973;1(1):1-30.

10. Solé RV, Ferrer R, González-García I, Quer J, Domingo E. Red queen dynamics, competition and critical points in a model of RNA virus quasispecies. J Theor Biol. 1999;198(1):47-59.

11. Furió V, Moya A, Sanjuán R. The cost of replication fidelity in an RNA virus. Proc Nat Acad Sci U S A. 2005;102(29):10233-10237.

12. Muller HJ. The relation of recombination to mutational advance. Mutat Res. 1964;106:2-9.

13. Worobey M, Holmes EC. Evolutionary aspects of recombination in RNA viruses. J Gen Virol. 1999;80(Pt 10):2535-2543.

14. Koonin EV, Dolja VV. Evolution and taxonomy of positive-strand RNA viruses: implications of comparative analysis of amino acid sequences. Crit Rev Biochem Mol Biol. 1993;28(5):375-430.
15. Gerrish PJ, Lenski RE. The fate of competing beneficial mutations in an asexual population. Genetica. 1998;102-103(1-6):127-144.

16. Miralles R, Gerrish PJ, Moya A, Elena SF. Clonal interference and the evolution of RNA viruses. Science. 1999;285(5434):1745-1747.

17. de la Torre JC, Holland JJ. RNA virus quasispecies populations can suppress vastly superior mutant progeny. J Virol. 1990;64(12):6278-6281.

18. Miralles R, Moya A, Elena SF. Diminishing returns of population size in the rate of RNA virus adaptation. $J$ Virol. 2000;74(8):3566-3571.

19. Imhof M, Schlötterer C. Fitness effects of advantageous mutations in evolving Escherichia coli populations. Proc Nat Acad Sci U S A. 2001;98(3):1113-1117.

20. Miller CR, Joyce P, Wichman HA. Mutational effects and population dynamics during viral adaptation challenge current models. Genetics. 2011;187(1):185-202.

21. Verbinnen T, Van Marck H, Vandenbroucke I, et al. Tracking the evolution of multiple in vitro hepatitis $\mathrm{C}$ virus replicon variants under protease inhibitor selection pressure by 454 deep sequencing. $J$ Virol. 2010;84(21):11124-11133.

22. Bantel-Schaal U, zur Hausen H. Characterization of the DNA of a defective human parvovirus isolated from a genital site. Virology. 1984; 134(1):52-63.

23. Laughlin CA, Myers MW, Risin DL, Carter BJ. Defective-interfering particles of the human parvovirus adeno-associated virus. Virology. 1979;94(1):162-174.

24. Palma EL, Huang A. Cyclic production of vesicular stomatitis virus caused by defective interfering particles. J Infect Dis. 1974;129(4):402-410.

25. Prince AM, Huima-Byron T, Parker TS, Levine DM. Visualization of hepatitis $\mathrm{C}$ virions and putative defective interfering particles isolated from low density lipoproteins. J Viral Hepat. 1996;3(1):11-17.

26. Horikami SM, Curran J, Kolakofsky D, Moyer SA. Complexes of Sendai virus NP-P and PL proteins are required for defective interfering particle genome replication in vitro. J Virol. 1992;66(8):4901-4908.

27. Perrault J. Origin and replication of defective interfering particles. Curr Top Microbiol Immunol. 1981;93:151-207.

28. Kirkwood TB, Bangham CR. Cycles, chaos, and evolution in virus cultures: a model of defective interfering particles. Proc Nat Acad Sci US A. 1994;91(18):8685-8689.

29. Marriott AC, Dimmock NJ. Defective interfering viruses and their potential as antiviral agents. Rev Med Virol. 2010;20(1):51-62.

30. Rao DD, Huang AS. Interference among defective interfering particles of vesicular stomatitis virus. J Virol. 1982;41(1):210-221.

31. Stumpf MP, Zitzmann N. RNA replication kinetics, genetic polymorphism and selection in the case of the hepatitis C virus. Proc Biol Sci. 2001;268(1480):1993-1999.

32. Navas S, Martín J, Quiroga JA, Castillo I, Carreño V. Genetic diversity and tissue compartmentalization of the hepatitis $\mathrm{C}$ virus genome in blood mononuclear cells, liver, and serum from chronic hepatitis $\mathrm{C}$ patients. J Virol. 1998;72(2):1640-1646.

33. Gretch DR, Polyak SJ. The quasispecies nature of Hepatitis C Virus: research methods and biological implications. In: Groupe Français d'études molécularies des hépatites (GEMHEP), Hepatitis C Virus: 2; Genetic Heterogeneity and Viral Load. Paris: John Libbey Eurotext; 1997:57-69. [English].

34. Cabot B, Martell M, Esteban JI, et al. Nucleotide and amino acid complexity of hepatitis $\mathrm{C}$ virus quasispecies in serum and liver. JVirol. 2000;74(2):805-811.

35. Wilke CO, Wang JL, Ofria C, Lenski RE, Adami C. Evolution of digital organisms at high mutation rates leads to survival of the flattest. Nature. 2001;412(6844):331-333

36. Wilke CO. Adaptive evolution on neutral networks. Bull Math Biol. 2001;63(4):715-730.

37. Codoñer FM, Darós JA, Solé RV, Elena SF. The fittest versus the flattest: experimental confirmation of the quasispecies effect with subviral pathogens. PLoS Pathog. 2006;2(12):e136.

38. Campo D, Dimitrova Z, Mitchell R, Lara J, Khudyakov Y. Coordinated evolution of the hepatitis C virus. Proc Nat Acad Sci U S A. 2008; 105(28):9685-9690. 
39. Sanjuán R, Moya A, Elena SF. The distribution of fitness effects caused by single-nucleotide substitutions in an RNA virus. Proc Nat Acad Sci US A. 2004;101(22):8396-8401.

40. Aguirre J, Lázaro E, Manrubia SC. A trade-off between neutrality and adaptability limits the optimization of viral quasispecies. J Theor Biol. 2009;261(1):148-155.

41. Earl DJ, Deem MW. Evolvability is a selectable trait. Proc Nat Acad Sci U S A. 2004;101(32):11531-11536.

42. Schuster P, Swetina J. Stationary mutant distributions and evolutionary optimization. Bull Math Biol. 1988;50(6):635-660.

43. Vignuzzi M, Stone JK, Arnold JJ, Cameron CE, Andino R. Quasispecies diversity determines pathogenesis through cooperative interactions in a viral population. Nature. 2005;439(7074):344-348.

44. Arbiza J, Mirazo S, Fort H. Viral quasispecies profiles as the result of the interplay of competition and cooperation. BMC Evol Biol. 2010;10:137.

45. de Visser JA, Rozen DE. Limits to adaptation in asexual populations. J Evol Biol. 2005;18(4):779-788.

46. Jain K, Krug J. Deterministic and stochastic regimes of asexual evolution on rugged fitness landscapes. Genetics. 2007;175(3):1275-1288.

47. Saakian DB, Biebricher CK, Hu CK. Phase diagram for the Eigen quasispecies theory with a truncated fitness landscape. Phys Rev E Stat Nonlin Soft Matter Phys. 2009;79(4 Pt 1):041905.

48. Crotty S, Cameron CE, Andino R. RNA virus error catastrophe: direct molecular test by using ribavirin. Proc NatAcad Sci U SA. 2001;98(12): 6895-6900.

49. Biebricher CK, Eigen M. The error threshold. Virus Res. 2005;107(2): 117-127.

50. Orr HA. The rate of adaptation in asexuals. Genetics. 2000;155(2): 961-968.

51. Stich M, Briones C, Manrubia SC. Collective properties of evolving molecular quasispecies. BMC Evol Biol. 2007;7:110.

52. Johnson T, Barton NH. The effect of deleterious alleles on adaptation in asexual populations. Genetics. 2002;162(1):395-411.

53. Arjan JA, de Visser M, Zeyl CW, Gerrish PJ, Blanchard JL, Lenski RE. Diminishing returns from mutation supply rate in asexual populations. Science. 1999;283(5400):404-406.

54. Elena SF, Lenski RE. Evolution experiments with microorganisms: the dynamics and genetic bases of adaptation. Nat Rev Genet. 2003;4(6): 457-469.

55. Clune J, Misevic D, Ofria C, Lenski RE, Elena SF, Sanjuán R. Natural selection fails to optimize mutation rates for long-term adaptation on rugged fitness landscapes. PLoS Comput Biol. 2008;4(9):e1000187.

56. Springman R, Keller T, Molineux IJ, Bull JJ. Evolution at a high imposed mutation rate: adaptation obscures the load in phage T7. Genetics. 2010;184(1):221-232.

57. Foster PL. Adaptive mutation: implications for evolution. Bioessays. 2000;22(12):1067-1074.

58. Cases-González C, Arribas M, Domingo E, Lázaro E. Beneficial effects of population bottlenecks in an RNA virus evolving at increased error rate. J Mol Biol. 2008;384(5):1120-1129.

59. Wainberg MA, Drosopoulos WC, Salomon H, et al. Enhanced fidelity of 3TC-selected mutant HIV-1 reverse transcriptase. Science. 1996;271(5253):1282-1285.

60. Metzgar D, Wills C. Evidence for the adaptive evolution of mutation rates. Cell. 2000;101(6):581-584.

61. Drake JW, Charlesworth B, Charlesworth D, Crow JF. Rates of spontaneous mutation. Genetics. 1998;148(4):1667-1686.

Virus Adaptation and Treatment

\section{Publish your work in this journal}

Virus Adaptation and Treatment is an international, peer-reviewed open access journal focusing on the study of virology, viral adaptation and the development and use of antiviral drugs and vaccines to achieve improved outcomes in infection control and treatment. The journal welcomes original research, basic science, clinical \& epidemiological
62. Cuevas JM, González-Candelas F, Moya A, Sanjuán R. Effect of ribavirin on the mutation rate and spectrum of hepatitis $\mathrm{C}$ virus in vivo. J Virol. 2009;83(11):5760-5764.

63. Drake JW, Holland JJ. Mutation rates among RNA viruses. Proc Nat Acad Sci U S A. 1999;96(24):13910-13913.

64. Vignuzzi M, Stone JK, Andino R. Ribavirin and lethal mutagenesis of poliovirus: molecular mechanisms, resistance and biological implications. Virus Res. 2005;107(2):173-181.

65. Airaksinen A, Pariente N, Menéndez-Arias L, Domingo E. Curing of foot-and-mouth disease virus from persistently infected cells by ribavirin involves enhanced mutagenesis. Virology. 2003;311(2):339-349.

66. Pfeiffer JK, Kirkegaard K. Ribavirin resistance in hepatitis $\mathrm{C}$ virus replicon-containing cell lines conferred by changes in the cell line or mutations in the replicon RNA. J Virol. 2005;79(4):2346-2355.

67. Young KC, Lindsay KL, Lee KJ, et al. Identification of a ribavirinresistant NS5B mutation of hepatitis C virus during ribavirin monotherapy. Hepatology. 2003;38(4):869-878.

68. Arnold JJ, Vignuzzi M, Stone JK, Andino R, Cameron CE. Remote site control of an active site fidelity checkpoint in a viral RNA-dependent RNA polymerase. J Biol Chem. 2005;280(27):25706-25716.

69. Sallie R. Replicative homeostasis III: implications for antiviral therapy and mechanisms of response and non-response. Virol J. 2007;4:29.

70. Sallie R. Replicative homeostasis: a fundamental mechanism mediating selective viral replication and escape mutation. Virol J. 2005;2:10.

71. Sallie R. Replicative homeostasis II: influence of polymerase fidelity on RNA virus quasispecies biology: implications for immune recognition, viral autoimmunity and other "virus receptor" diseases. Virol $J$. 2005;2:70.

72. Hopfield JJ. Kinetic proofreading: a new mechanism for reducing errors in biosynthetic processes requiring high specificity. Proc Nat Acad Sci US A. 1974;71(10):4135-4139.

73. Simon B. A stochastic model of evolutionary dynamics with deterministic large-population asymptotics. J Theor Biol. 2008;254(4):719-730.

74. Bukh J, Pietschmann T, Lohmann V, et al. Mutations that permit efficient replication of hepatitis C virus RNA in Huh-7 cells prevent productive replication in chimpanzees. Proc Nat Acad Sci U S A. 2002;99(22):14416-14421.

75. Mueller LD, Ayala FJ. Trade-off between r-selection and K-selection in Drosophila populations. Proc Nat Acad Sci U S A. 1981;78(2): 1303-1305.

76. Giraud A, Matic I, Tenaillon O, et al. Costs and benefits of high mutation rates: adaptive evolution of bacteria in the mouse gut. Science. 2001;291(5513):2606-2608.

77. Sevilla N, Ruiz-Jarabo CM, Gómez-Mariano G, Baranowski E, Domingo E. An RNA virus can adapt to the multiplicity of infection. J Gen Virol. 1998;79(Pt 12):2971-2980.

78. Rosenberg SM, Thulin C, Harris RS. Transient and heritable mutators in adaptive evolution in the lab and in nature. Genetics. 1998;148(4):1559-1566.

79. Alter HJ, Conry-Cantilena C, Melpolder J, et al. Hepatitis C in asymptomatic blood donors. Hepatology. 1997;26(3 Suppl 1):29S-33S.

80. Alter HJ, Seeff LB. Recovery, persistence, and sequelae in hepatitis C virus infection: a perspective on long-term outcome. Semin Liver Dis. 2000;20(1):17-35.

81. Sanjuán R. Mutational fitness effects in RNA and single-stranded DNA viruses: common patterns revealed by site-directed mutagenesis studies. Philos Trans R Soc Lond B Biol Sci. 2010;365(1548):1975-1982.

\section{Dovepress}

studies, reviews \& evaluations, expert opinion and commentary, case reports and extended reports. The manuscript management system is completely online and includes a very quick and fair peer-review system, which is all easy to use. Visit http://www.dovepress.com/ testimonials.php to read real quotes from published authors. 